\title{
Uniaxial equivalent of stress and stress intensity factor in Mode I crack opening for fatigued metals subjected to multi parametric external loading
}

\author{
Andrey Shanyavskiy ${ }^{1,2, *}$ \\ ${ }^{1}$ Aviation Register of the Russian Federation, 14142, Sheremetyevo-1 Airport, Moscow Region, Russia \\ ${ }^{2}$ Moscow Aviation Institute (National Research University), 125993, Volokolamskoe street, 4, Moscow, Russia
}

\begin{abstract}
The principles of physical mesomechanics and synergetics, provides for a measure of energy involved in the ability of a material to resist deformation and fatigue cracking used for introducing a concept of equivalent stress and stress intensity factor in Mode I crack opening of fatigued metals. Acquired under the standard test conditions, the curves of unidirectional tension and those of fatigue duration and of crack-growth kinetics show themselves applicable as universal master-diagrams descriptive of the material behavior though under multiparametric loading. Specimen patterns of fracture, revealed by the test pieces of metallic alloys given to asymmetrical biaxial loading or multiparametric loading, passed careful research. In the biaxial case, this research facilitated finding of the view of the correction function for the level of equivalent stress and, in the multi-axial case, employing of the above subject ideas to ascertain the very level of equivalent stress.
\end{abstract}

\section{Introduction}

In terms of physical mesomechanics or of synergetics, the evolution behavior of externally affected metallic materials involves a sequence (cascade) of selforganized and self-consisted energy-storing processes of differing scale levels [1-3]. It is a property of metallic materials to meet an external attack by starting a cascade of the evolution processes, each cascade pattern inherently peculiar to a particular metal. Those processes do (or do not) arise depending not on the mode of the external energy supply but on whether this supply does take place at all; in fact, the processes answer any mode of external attack and arise on a scale consistent with the growth of the material volume to absorb supplied energy [2]. As the energy intensity of cyclic loading begins to grow, the test metal first absorbs this energy free of fracture effects, i.e., preserves its integrity by switching on still new intrinsic mechanisms of storing energy and thereby avoids creation of new free surface. The greater a scale level the more complex the energy storing process ensuring the metal soundness (integrity): indeed, the volume $\Delta V$, to store in the energy amount $\Delta W$, is greater in the latter case [2, 3].

In real service conditions, external action by cyclical loading on an engineering structure is likely to happen at various combinations of the cycle parameters. Therefore, we may talk about multiparametric field of external attack on a metallic material [3]. In addition, it may be that the operating fracture mechanisms follow one another in a sequence, varying with time (i.e., with growing amount of damage) and depending on the sequence in that greater and smaller energy per loading cyclic is supplied to the test structure. Consequently, one can characterize the variety of loading conditions, using quite a number of parameters, including those reflecting the metal reaction to an external attack.

They most often consider in separate, i.e., for each of the single parameters, those effects that those parameters have on the process of building up damage. For instance, they look in separate into the effects of temperature, stress ratio, cycling frequency, etc., on material durability and crack propagation rate. In some instances, either of the two latter characteristics may be a subject examined as depending jointly on temperature and stress ratio [6]. Nevertheless, for however differing conditions of external action, they employ always the same structural characteristics of the material reaction in order to describe the process of building up material damage at the stage of crack nucleation and propagation. Typically, they consider the ordered cascade of dislocationstructure types, which sequentially replace one another [4], the crack-propagation rate, and/or the elements of the fracture-surface morphology, e.g., fatigue striation patterns relevant to various stages of crack propagation under various loading conditions, etc. [3].

Metallic materials respond to multiparametric cyclic loading by absorbing energy in an adequate way. Such a 
response turns out similar to that under the standard conditions of uniaxial loading provided the mode of final deformation remains the same for the varying multiparametric attacks. One can see the similarity of this kind between the cases of monotonic uniaxial tension and cyclic loading showing the normal (Mode I) crack-opening behavior [3].

We may, therefore, describe a material behavior with the use of a single approach to the idea of the density of deformation energy $[5,6]$ and of the density of fracture energy $[3,7]$; in so doing we take, for an integrated characteristic, the parameters of the material reaction to an external affection for any combination of the loadingcycle parameters. Here, the critical requirement is to ensure that, under the external action of an arbitrary kind, material stretching would dominate at the singlepath loading and Mode I normal crack opening, at the cyclic loading.

\section{Durability}

In the materials under cyclic loading, damage of various scales builds up, to agree with the tensile stress-strain diagram [2, 3, 7]. In metallic materials, the built up damage forms in [8] and beneath [9] the surface layer, and the oncoming of the limiting state, with its concomitant initiation and propagation of a fatigue crack, takes a period depending on the level of the applied stress; characteristic of the limiting state is the number $N_{\mathrm{f}}$ of cycles to failure. Each of the loading cycles brings in an energy density $\mathrm{d} W / \mathrm{d} V$. At various scale levels, the latter quantity is characteristic of the limiting state of a material in terms of the number of cycles that the material can resist until the fracture of all volume $\Delta V$.

Let us consider the stress factor in its dimensional form $\mathrm{kg} / \mathrm{mm}^{2}$. Having multiplied this ratio by $\mathrm{mm} / \mathrm{mm}$, we come about a dimension, $\mathrm{kg} \times \mathrm{mm} / \mathrm{mm}^{3}$, peculiar to the deformation work $(\mathrm{d} W / \mathrm{d} V)=\sigma_{\mathrm{e}}$ of the unite volume of a material, i.e., come about the density of strain energy. With any method of affecting a material, the attained $\varepsilon_{\mathrm{j}}$ value relates to the only magnitude $(\mathrm{d} W / \mathrm{d} V)_{\mathrm{ej}}$ of energy density or the only level $\sigma_{\mathrm{ej}}$ of equivalent stress.

The idea that, in metals, energy builds up on the differing scales makes it necessary, when describing the metal behavior, to consider the bifurcation diagram [9] adopted for the specified standard experiment conditions. We use preserved the entire test parameters of an external cyclic influence, and only vary applied uniaxial tensile stress, setting it different for different test specimens.

Based on the concept of the equivalent tensile stress $[3,7]$, one can reduce the earlier drawn bifurcation diagram of fatigue curve to a general form of fatigue durability versus equivalent stress or strain-energy density, Fig. 1. Equality in the fatigue durability provides for a criterion that the etalon test conditions (relevant to the etalon fatigue diagram) are equivalent to the test conditions in a multiparametric space, i.e., relevant to the master diagram.
A metallic material achieves its limiting state of crack nucleation as soon as the strain energy builds up to the critical amount, which is independent of the applied stress level. Therefore, one can accept for every scale that

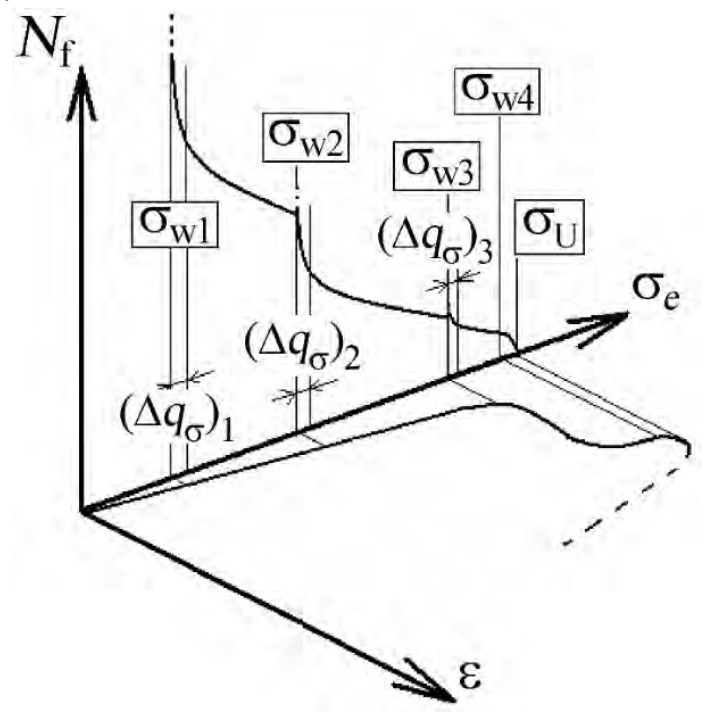

Fig. 1. A bifurcation bearing plot $N_{\mathrm{f}}-\sigma_{\mathrm{e}}$ (schematic) of metal fatigue, drawn in agreement with the diagram $\sigma_{\mathrm{e}}-\varepsilon$ of tension tests and in terms of "equivalent stress level $\sigma_{\mathrm{e}}$ " or "strain energy density $\mathrm{d} W / \mathrm{d} V$ '. The bifurcation ranges $\Delta q_{\sigma i}$ of the transitions to micro- or nano- $\left(\sigma_{\mathrm{w} 1}-\sigma_{\mathrm{w} 2}\right)$, meso- $\left(\sigma_{\mathrm{w} 2}-\sigma_{\mathrm{w} 3}\right)$, and macro-scale $\left(\sigma_{\mathrm{w} 3}-\sigma_{\mathrm{w} 4}\right)$ levels of fracture [9].

$$
N_{\mathrm{f}}(\Delta W / \Delta V)=\text { const. }
$$

In terms of the equivalent stress, Eq. (1) coincides qualitatively with the well-known equality from Wöhler [10],

$$
\sigma_{\mathrm{e}}^{\mathrm{m}} N_{\mathrm{t}}=C_{\mathrm{f}}
$$

Equation (2) involves the exponent $m_{\mathrm{f}}=2$ as long as $\Delta W / \Delta V=\sigma_{\mathrm{e}}{ }^{2} /(2 E)[8,9]$, e.g., for the macro elastic highcycle fatigue behavior of metallic materials. Actually, the exponent $m_{\mathrm{f}}$ magnitude differs significantly from two to demonstrate that, in fact, the consumed amount of strain energy is characteristic of different scales of the material behavior. In a metallic material the defects generated by cyclic loading build up predominantly in the surface layer of a test piece; accordingly, the test piece consumes the strain energy supplied by cyclic loading, and crack nucleation occurs in the sites of particularly high concentration of the defects. Therefore, one would be wrong employing a macroscopic approach in order to describe an operative mesoscopic pattern (building up damage) of material behavior.

The surface layer differs significantly from the interior part of the material body [8]. In the surface layer, damage builds up of both the macroscopic and mesoscopic scales. The $\Delta W / \Delta V$ magnitude is a macroscopic characteristic of metal deformation all over the test-piece body, in accordance with the tensile stressstrain diagram. Hence, to ascertain the whole number of cycles to failure, one has to measure the $\mathrm{d} W / \mathrm{d} V$ magnitude as related to the scale level of the 
mechanisms that control effects of building up damage, including those taking place in the surface layer of the test sample.

Let us regard the mesoscopic scale of the strainenergy magnitude, which controls the surface cracknucleation effects under high-cycle fatigue, and estimate how much this magnitude deviates from that inferred from Eq. (1). In so doing, assume that the limiting state of a material, described by Eqs. (1) and (2), requires the same level of the limiting energy (characteristic of the breaking point) for both macroscopic and mesoscopic scales; then, the local energy density at the loading part of the cycle is $m_{\mathrm{f}} / 2$ times as big as the $\mathrm{d} W / \mathrm{d} V$ value, inferred from the tensile stress-strain graph.

Thus, for each of the scales, equations descriptive of the relation of the value of test-piece durability to the applied stress or to the strain-energy density, expressed in terms of "equivalent stress", get the view as presented in [9].

One should particularly mind that, in case of cyclic loading, another parameter, the stress ratio $R=\sigma_{\min } / \sigma_{\max }$, appears. Its effect on the material behavior is similar to that of the deviations of equivalent stress or of strainenergy density; likewise, some of the parameters influence the test-piece behavior under tension.

\section{Crack Propagation}

K.J. Miller [11] first suggested to regard the magnitudes of crack-growth rate and the size of a crack-tip zone as an integrated characteristic of a material reaction to the propagation of fatigue-cracks starting from the test-piece surface and having the crack front of various shape. For two differing test conditions, the stress-intensity factors $K_{\mathrm{I} i}$ and $K_{\mathrm{I} j}$ will be equal to one another as long as the two crack-growth rates or (which is the same) the sizes of the two crack-tip plastic zones are, respectively, also equal to one another, but crack lengths $a_{i}$ and $a_{i+1}$ will be different for two compared cases. The two crack lengths $a$ in the ratio $a_{i} / a_{i+1}$ correspond to the above mentioned two different test conditions; and the ratio figure indicates how much the crack length must change, with the test conditions, to thereby keep unchanged the crackgrowth rate (or the plastic-zone size).

The above criterion was a subject of investigation as applied to the case of uniaxial or biaxial tension (also to a tension-compression case) of flat models [11]. A significant requirement is that one supports the normal (Mode I) crack-opening behavior and similarity of the fracture mechanisms for the two compared test conditions. We introduce a correction $f\left(a_{i} / a_{i+1}\right)$ for the crack length, which, in fact, is a dimensionless correction for the stress ratio $\lambda=\sigma_{1} / \sigma_{2}$ in the case of biaxial loading, where $\sigma_{1}$ is the stress of uniaxial tension and $\sigma_{2}$ is the stress applied normal to $\sigma_{1}$.

Another approach, which helps one to universally describe the growth behavior of a fatigue crack in a metallic material, makes use of the concept of effective stress-intensify factor $\Delta K_{\text {eff }}$ [12], its magnitude inferred from the effect of incompletely closed fatigue crack (crack lips). McClung [13] found out that the three kinetic curves (patterns of biaxial-tension or of tensioncompression included) plotted as functions of stress ratio, are equidistant with respect to one another and can be reduced to a single curve, as applied to the specified standard (adopted) conditions of the experiment.

As a matter of fact, the effect of incompletely closed crack lips can vary in its nature, i.e., in the mechanisms that control the crack-tip behavior [14]. Owing to such an uncertainty, the similarity might be lacking between the growth patterns of the fatigue cracks, despite still maintained equality between the magnitudes of the above mentioned effective stress-intensity factor; hence, the prospects become uncertain of employing the latter as a universal characteristic of a multiparametric affection.

Now we are going to demonstrate greater universality of a criterion based on the idea of the density of fracture energy.

Imagine a fatigue-crack front line as a nonstretchable string moved along two parallel strings by the action of a force $F$ (Fig. 2). In proportion to the string displacement (crack increment), the work $\mathrm{d} W=F \mathrm{~d} a$ is being done.

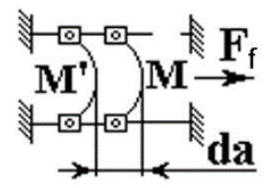

a)

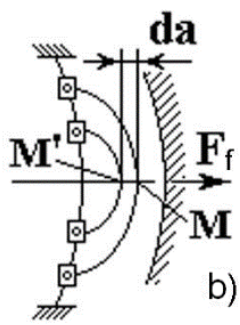

Fig. 2. The crack frontline (schematic) in the form of a stretchable string for the cases of (a) through fatigue crack or (b) surface fatigue crack; the frontline shifts, moved by a force applied to a material point $\mathrm{M}$, in the crack-growth direction.

Returning to the pattern of crack, we mind that the crack lengthens owing to the event of breaking down some volume $\mathrm{d} V$ of the material within the limits of the plastic zone. Having considered some area ds, normal to the crack-growth direction, we multiply and divide by $\mathrm{d} s$ the right part of the above-derived relationship to have a result as follows,

$$
\mathrm{d} W=(F / \mathrm{d} S) \mathrm{d} a \mathrm{~d} s=\sigma_{\mathrm{f}} \mathrm{d} V, \text { or } \mathrm{d} W / \mathrm{d} V=\sigma_{\mathrm{f}} .
$$

Thus obtained stress magnitude is proportional to the total effect of the combined attacks terminated at the crack increment, i.e., proportional to the equivalent stress $\sigma_{e}$; one should also mind that, for the same stress, the crack-growth rate is the higher the less the elasticity modulus of the material. One can take account of this interrelation using the value of strain revealed by the loaded test piece. Then we finally have

$$
(\mathrm{d} W / \mathrm{d} V)_{\mathrm{f}}=\left(1-v^{2}\right) \sigma_{\mathrm{e}}^{2} / E .
$$

In Eq. (4), we take into account the Poisson's effect of the transition between the two mutually normal stress vectors. This equation is descriptive of the crackincrement in a fatigue process; here, equivalent stress $\sigma_{\mathrm{e}}$ represents the density of fracture energy $(\mathrm{d} W / \mathrm{d} V)_{\mathrm{f}}$ 
peculiar to the crack-increment event in a material during a loading cycle and can be estimated by the next equation:

$$
\sigma_{\mathrm{e}}=\sigma_{1} F\left[f\left(\mathrm{X}_{1}\right), f\left(\mathrm{X}_{2}\right), \ldots, f\left(\mathrm{X}_{i}\right)\right]
$$

In Eq. (5), $\sigma_{e}$ is the value of equivalent stress characteristic of an integrated reaction of a material on the external multiparametric attack, $F\left[f\left(\mathrm{X}_{1}\right), f\left(\mathrm{X}_{2}\right), \ldots, f\left(\mathrm{X}_{i}\right)\right]$ is the correction function for the effect of the parameters $\mathrm{X}_{i}$ on the deformation behavior of a material, and $\sigma_{1}$ is the stress of standard uniaxial tension representing one of the constituents of the multiparametric attack for the case of $\left[f\left(\mathrm{X}_{1}\right), f\left(\mathrm{X}_{2}\right), \ldots, f\left(\mathrm{X}_{i}\right)\right]=1$.

Bearing in mind Eq. (3), we may suggest the way of the universal description of fatigue cracks propagation; in so doing, we employ the entities of crack-growth rate and of fatigue-striation spacing $\delta_{i}$ in the relationships for different types of Al, Fe, Ti based alloys [3], [7], [1517].

Thus, the concept of equivalent stress provides for a single approach to the problem of having described the behavior of metallic materials in terms of a universal characteristic, the energy density, applicable to whether the fracture or the deformation events.

Let us now ground on the principles of physical mesomechanics and synergetics to see, from examples, how efficient is to employ the above concept when producing a universal description of the behavior of metallic materials under multiparametric cyclic loading.

\subsection{Example 1}

Shanyavsky et al. [15] reported on the tests of cruciform test specimens (imitational model) by cyclic biaxial tension, with the concurrently changed cycle parameters, as well as under compression-tension conditions with a diverse stress ratio. The 5-mm thick specimens, made of D16 aluminum-based alloy, passed the tests in the range $-1<\lambda<1$ of principle-stress ratio $\left(\lambda=\sigma_{1} / \sigma_{2}\right)$ and in the range 0.1 to 0.8 of the stress ratio $R$.

The variations of the cycle parameters involved correction functions found from a relationship

$$
C_{I s 0} / C_{I s i}=F(R, \lambda) .
$$

The authors [15] acquired the $C_{I S 0}=\left(1-v^{2}\right) /\left(8 \pi \sigma_{0.2} E\right)$ magnitude of relation (6) as applied to the standard test conditions when crack-growth rate has proportion to the $\left(K_{\mathrm{e}}\right)^{2}$ of stress equivalent factor. They inferred the $C_{I s i}$ magnitude from the test results, including the measured crack-growth rates and striation spacing for various combinations of the value $R$ and the ratio $\lambda$ of principle stresses when kinetic curves have equidistant position of one to another one.

They found out that, throughout the said range of the combined loading-cycle parameters, the curves of fatigue-crack growth remain equidistant with respect to the one and another for the crack-growth rate or for striation spacing [16]. The same (equivalent) crackgrowth kinetics persisted all over the examined range of variously combined parameters $R$ and $\lambda$ of the loading cycle. For the aluminum-based D16T alloy, the authors [16] determined the magnitudes of equivalent stress (with respect to the stress used in the uniaxial cyclic loading). They found out that, in the mentioned range of the parameters $R$ and $\lambda$, the correction functions for the mentioned magnitudes of the equivalent stress take the view of polynomials as follows for the striation spacing $\delta$ :

$$
\begin{aligned}
& F^{2}\left(\lambda_{\sigma}, R\right)_{\delta}=0.953-0.547 \lambda_{\sigma}-0.099 \lambda_{\sigma}^{2}+0.588 R+ \\
& +0.839 R \lambda_{\sigma}-0.201 R \lambda_{\sigma}^{2}-5.184 R^{2}-0.329 R^{2} \lambda_{\sigma}+ \\
& \quad+2.091 R^{2} \lambda_{\sigma}^{2}+3.855 R^{3}+0.08 R^{3} \lambda_{\sigma}-2.093 \lambda_{\sigma}^{2} R^{3}
\end{aligned}
$$

It follows from Eq. (7) that, in the case of multiparametric cycling, nonlinear functions describe the effect of the cycling parameters on a material behavior and indicate to the maintained law of similarity between the fracture mechanisms or between the crack-growth kinetics.

\subsection{Example 2}

The author [17] examined the cases of in-service failure of the longerons of helicopter blades (Mi-family helicopters) manufactured from Al-alloy AVT. In service, those longerons experience the complicated multiparametric effects of tension, torsion, and bending, as well as of one-atmosphere excessive internal pressure. A pressure gage, built in the longeron, responds to depressurization, thus warning about an occurred discontinuity.

Fatigue cracking, ordinarily starting from various material defects, might be a result of loading over a limit preset by a designer. Therefore, the author's aim had to answer if the in-service stresses did not exceed a designed limit.

On the strength of that, the author performed a set of fractographic examinations, the special bench tests of the longeron fragments, as well as the standard tensile tests of the specimens cut out of the longerons. The author answered the question about the in-service loading pattern of the longerons by having performed a twostage research. At the beginning, the author examined the specimens that passed the tests by bi-parametric loading: by tension and torsion. Fractographically, the specimens showed fatigue striations formed all along the crack-growth direction; this finding confirmed the maintenance of the similarity law for the fracture mechanism in a wide range of the loading-cycle parameters as concerns the stress ratio $R$ of the loading cycle and the angle $\omega$ of torsion. Of the correction functions $F(R, \omega)$ involved in the relationship

$$
C_{I s 0} / C_{I s i}=F(R, \omega),
$$

a magnitude $C_{I s i}$ answers the test data, including the measured values of crack-growth rate and of striation spacing for the variously combined magnitudes of stress ratio $R$ and torsion angle $\omega$. 
The test data, including those on the crack-growth rates, show that, plotted as the functions of the stressintensity factor $K_{\mathrm{I}}$, all the curves of the cracking kinetics appear equidistant with respect to one another. A correction function had a view [17],

$$
F_{i}^{2}(\omega)=A_{1}+A_{2} \omega+A_{3} \omega^{2}+A_{4} \omega^{3}+A_{5} \omega^{4}
$$

for each of the stress ratio (with $R=$ const) with different $A_{i}$ constants.

Using the values of coefficients for unified fatigue crack growth description [17] made it possible to reduce all the curves to a single one, related to an equivalent stress-intensity factor $K_{\mathrm{e}}$ (Fig. 3).

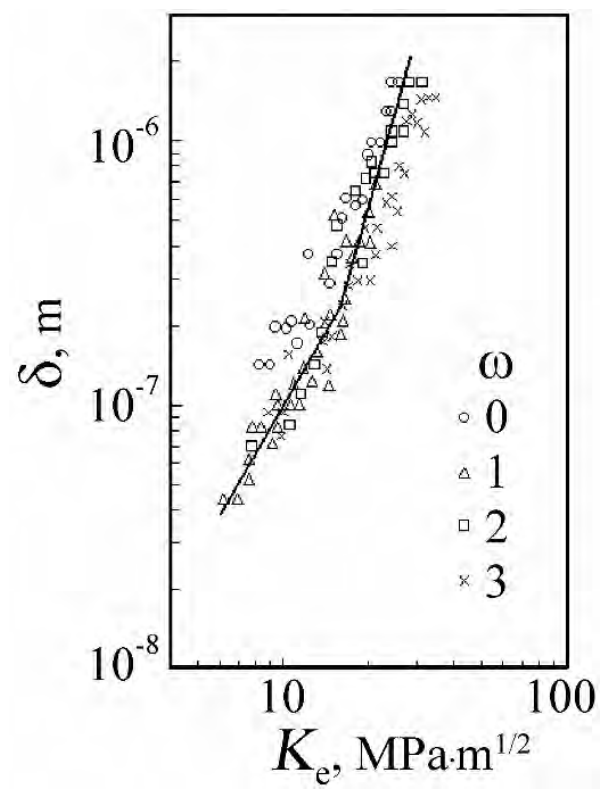

Fig. 3. The test data, on the rectangular specimens of AVT alloy, obtained at various torsion angles $\omega$ and a magnitude $R=0.1$ of stress ratio. A single unified curve of crack-growth kinetics (solid line) as compared with the superimposed data points of striation spacing $\delta$, plotted as functions of the stressintensity factor $K_{\mathrm{e}}=K_{\operatorname{Imax}} F(R, \omega)[17]$.

At the second stage of these investigations, the author employed the formulas of fracture mechanics to have calculated the levels of equivalent stress. The calculations concerned various crack lengths, various cross sections of rotor blades, and various helicopter units, i.e., covered different loading conditions typical of the longeron in service.

Thus acquired, the data turned out to agree with those obtained, by designers, in the framework of traditional calculation techniques with 15 to $20 \%$ calculation accuracy.

\subsection{Example 3}

The helicopter V-3 passed through a severe flying accident after 90 -hour operation in service. The accident circumstances and all structure elements of the helicopter were examined to find out that the braking events mostly began from the pitch levers of the rotor.
And one of the levers (No. 4) appeared broken by fatigue Fig. 4.

The fracture began from a bottom ledge of the Ishaped part of the lever manufactured from Fe-based alloy with $1800 \mathrm{MPa}$ of ultimate tensile stress. In its starting zone, the fracture looked quite smooth and free of any distinct plastic-shear lips. The fracture path is illustrated by the sequence of fracture micrographs in Fig. 5. From the micrographs one can see the fracture surface cracked and showing (locally) fatigue striations, whose spacing achieved $5 \times 10^{-7} \mathrm{~m}$ quite close to the fracture-origin zone. Such a fracture pattern persisted to the crack lengths of 5-7 $\mathrm{mm}$. As the crack length grew further the cracking effects of the fracture surface became more extensive.

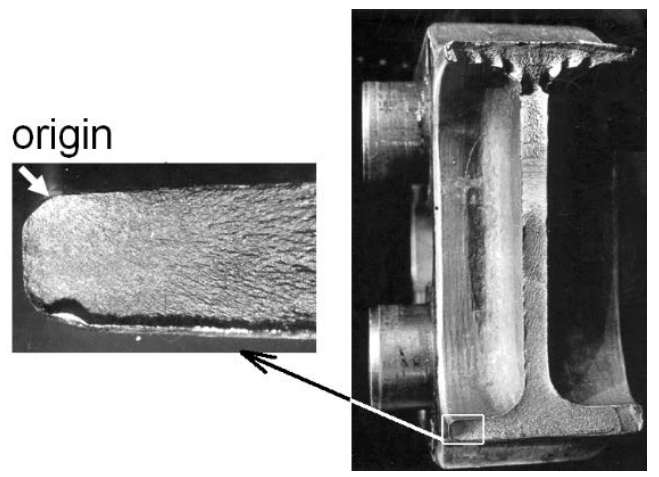

Fig. 4. View of the lever fracture surface with indication area of crack origination.

The most important here is the problem of crackgrowth period and equivalent-stress level; both were estimated based on the single curve of fatigue kinetics based on discussed concept of equivalent value of stress intensity factor.

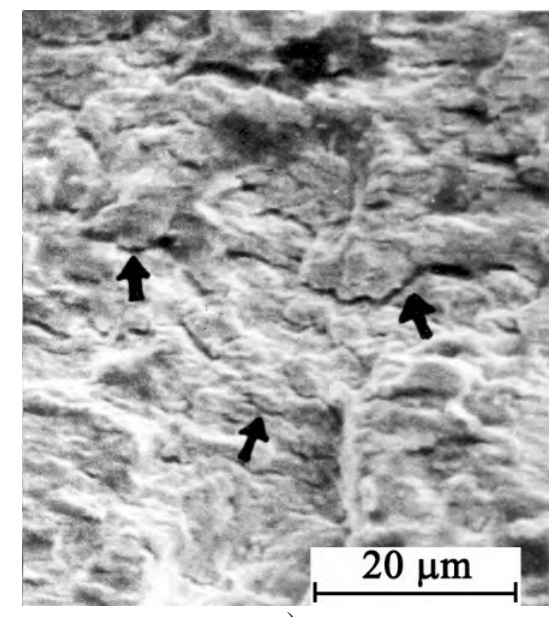

a) 


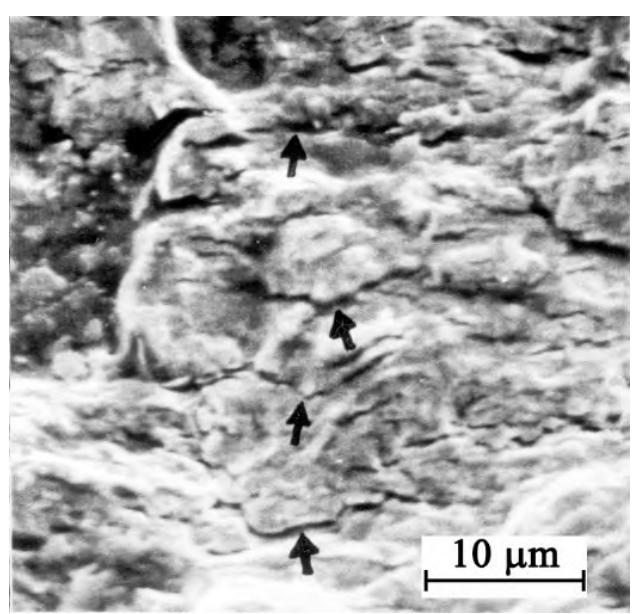

b)

Fig. 5. Areas with cracks (indicated by arrows) and fatigue striations (a), (b) on the different distance from the origin.

The average crack-growth rate approximates $1.2 \times 10^{-6}$ $\mathrm{m}$ per loading cycle at the fracture stage where this rate is proportional to the value of equivalent stress-intensity factor to the power of four in accordance with unified kinetic curve for Fe-based alloy [3], [7]. In the subject case the distance of stable crack growth measured about $30 \mathrm{~mm}$. So we can estimate that the crack-growth period took at least 30000 loading cycles. If, instead, we had used the smallest crack-growth rate of $5 \times 10^{-7} \mathrm{~m}$ per loading cycle, the greatest value of crack-growth period would achieve 70000 cycles. As follows from the data of in-flight and bench tests, a basic revolution frequency (4 $\mathrm{Hz}$ ) of the rotor makes a basic loading frequency of the propeller-pitch levers. This means that the crack propagation took 2 to 5 hours and could come to the critical crack length during the last flight.

Judging from the designed stress level - $50 \mathrm{MPa}-$ and the portion $-80 \%$ - of fatigue fracture in the total cross-section area, the starting (crack-nucleation) and final (end-rupture) levels of equivalent stress differed appreciably. The threshold stress-intensity factor is at least $10 \mathrm{MPa} \mathrm{m}{ }^{1 / 2}$ for the high-strength subject steel that was used for manufactured structure. This value predicts that an initial defect (or inherited crack) not shorter than $10 \mathrm{~mm}$ is to exist to make possible initiation of a fatigue crack at the stress level of $50 \mathrm{MPa}$. Yet no initial crack was revealed at all in the discussed lever. Therefore, the nucleation and growth of the fatigue crack should have occurred at a substantially greater stress level, which we estimated based on the following concepts.

As follows from the single fatigue-kinetics curve, an equivalent value, about $50 \mathrm{MPa} \mathrm{m} \mathrm{m}^{1 / 2}$, of stress intensity factor is required to maintain the growth rate of fatigue crack near $5 \times 10^{-7} \mathrm{~m}$ per loading cycle. Such a growth rate was measured in the region about $3 \mathrm{~mm}$ ahead of the crack-origin site. At this stage, the steady-state growth of the crack occurred and we could analyze this growth like in the case of steady-state growth of a through crack in a specimen of rectangular cross-section. The crack here was assumed to reach far enough from its origin site, though its length remained rather small as compared with the general dimension of the lever cross-section.
Therefore, we estimated the equivalent stress level using the following simple relationship,

$$
\sigma_{\mathrm{e}}=K_{\mathrm{e}} / \sqrt{\pi a}=50 / \sqrt{3.14 \times 3 \cdot 10^{-3}} \geq 500 \mathrm{MPa}
$$

This stress value is somewhat underestimated because we neglected here certain corrections, including those for the crack-front geometry. Moreover, we only considered the smallest values of crack-growth rate and equivalent stress-intensity factor.

In parallel, flat specimens, specially cut out of similar levers, were tested bend-loaded. Judging from the test results, the fracture morphology revealed by the broken lever was characteristic of cracking at a high nominal tensile (uniaxial) stress and a high stress-intensity factor. This finding, which showed again to the efficiency of the unified fatigue-kinetics curve as used in calculations of an equivalent stress, made it clear that one still should reveal the way in that the loads applied to the lever in service became greater than predicted by design.

Based on the discovered stress equivalent value more than $500 \mathrm{MPa}$, special (short-term) in-flight tests were conducted with strain-gages installed in the crackinitiation zone. The test conditions were applied with due account of the fact that the subject helicopter operated being 2-ton overloaded, which should have influenced somehow the operation conditions of all its structure elements.

The in-flight tests showed that stresses as high as 700 $\mathrm{MPa}$ can really arise in the propeller-pitch levers of a helicopter overloaded to the level mentioned above. This finding conformed very well with the above stresscalculation results, acquired by processing of the qualitative-fractography data in accordance with the concept of single fatigue-kinetics curve. (Besides, thus estimated figure of the crack-growth period appeared quite consistent with the documented in-flight time-tofailure of the lever.) Done based on those results, the inflight tests appeared well programmed, properly performed, and informative. The test results definitely confirmed that the total overload of the helicopter was what actually initiated and controlled fatigue failure of the lever and, consequently, caused this severe flight accident of the vehicle.

The three presented examples show true the abovediscussed concept of equivalent stress, the latter acquired from the material reaction to an external multiparametric attack; the concept conforms to the well-known alternatives of the fracture-mechanical approach to the problem of estimating the stress level of metallic constructions.

\section{Conclusions}

In terms of physical mesomechanics and synergetics, a concept of equivalent stress, put forward in the present effort, considers such a stress as a physical characteristic of the material behavior and of the energy stored in a unite material volume under the dominating effects of single-acting and/or cyclic tension. 
The present research snows a cyclic lifetime (durability under cyclic loading) as informing on the material reaction to a multiparametric attack; at whichever scale level, the durability figure strongly depends on the portions of strain energy supplied with each loading cycle. The magnitude of energy density per loading cycle, as available from the tensile stress-strain diagram, i.e., relating to a macro-scale level, cannot be characteristic of the material durability if in the range of high-cycle fatigue. Indeed, in this range, one should regard the said energy density as related to the surface layer, in that fatigue cracks nucleate, i.e., to the mesoscale level of deformation.

At the stage of fatigue-crack propagation, an ordered sequence (cascade) of the fracture mechanisms shows itself; each of those mechanisms is uniquely relative to one of the respective scale levels of each material reaction against the multiparametric attack and, therefore, furnishes the way for having identified a level of equivalent stress.

Judging from data on experimental bi-parametric attacks on a material as well as from the findings brought in by the analysis of the broken parts of real structures subjected to multiparametric attacks, the estimations of the level of equivalent stress are proving reliable.

This study received a Supporting Grant from the Russian Scientific Foundation (Project No. 16-19-10376).

\section{References}

1. V.S. Ivanova, Synergetics, Strength and Fracture of Metallic Materials (Cambridge Intern. Science Publ., 1998)

2. V.E. Panin, V.E. Egorushkin, A.V. Panin, A.G. Chernyavskiy, Fiz. Mesomech., 19, 1 (2016)

3. A.A. Shanyavskiy, Modeling of Fatigue Failure of Metals. Synergetics in Aviation (Ufa, Monograph, 2007)

4. N.A. Koneva, D.V. Lychagin, L.A. Teplyakova, E.V. Kozlov, Theory and Experiment in the Study of Disclinations, 116-126 (Leningrad, PhysicalTechnical Institute, 1986)

5. G.C. Sih, X.S. Tang, Theor. Appl. Fract. Mech., 43, 2 (2005)

6. P. Lazzarin, F. Berto, F.J. Gomez, M. Zappalorto, Int. J. Fatigue, 30, 8 (2008)

7. A.A. Shanyavskii (1998) PROBAMAT-21st Century: Probabilities and Materials, 46, 11-44 (Dordrecht, Springer, 1998)

8. V.E. Panin, Fiz. Mesomech., 2, 6 (1999)

9. A.A. Shanyavskiy, Fiz. Mesomech., 17, 6 (2014)

10. A. Wöhler, Zeitschr. für Bauwesen, 13, pp. 233-258 (1863)

11. K.J. Miller, Met. Sci., 11, 8-9 (1977)

12. W. Elber, Damage Tolerance in Aircraft Structures, pp. 230-242 (ASTM STP 486, Philadelphia, 1971)
13. R.C. McClung, Fatigue Fract. Eng. Mater. Struct., 12, 5 (1989)

14. R.O. Ritchie, Mater. Sci. Eng.: A, 103, 1 (1988)

15. A.A. Shanyavsky, E.F. Orlov, M.Z. Koronov, Fatigue Fract. Eng. Mater. Struct., 18, 11 (1995)

16. A. Shanyavskiy, Engng Fract. Mech, 78, 8 (2011)

17. A.A. Shaniavski, Fatigue Fract. Eng. Mater. Struct., 19, 9 (1996) 